\title{
El sistema de coagulación y COVID-19
}

\author{
The coagulation system and COVID-19
}

\author{
Jorge A. Ríos Pérez
}

\section{Resumen}

Las anormalidades de la coagulación y el desarrollo de complicaciones trombóticas por COVID-19 parecen estar desencadenadas por una inflamación descontrolada, y ser clínicamente diferentes de otras entidades como la coagulación intravascular diseminada. Los mecanismos subyacentes incluyen la disfunción endotelial e interacciones entre el sistema inmunológico y el de coagulación. Los eventos trombóticos parecen ser más frecuentes en pacientes críticos con COVID19. Por ende, una estrategia distinta para el manejo de la trombosis asociada a COVID-19 podría ser necesaria. Sin embargo, son necesarios ensayos clínicos para determinar las estrategias más adecuadas en el manejo de los eventos trombóticos asociados a COVID-19.

Palabras clave: COVID-19, desórdenes de la coagulación, trombosis.

\section{Abstract}

The development of thrombotic complications in COVID-19 appears to be driven by uncontrolled inflammation, and it is clinically different from other conditions such as disseminated intravascular coagulation. The underlying mechanisms seem to be related to endothelial dysfunction and feedback loops between the immune system and the coagulation system. Thrombosis seems more frequent in critically ill patients with COVID-19. This suggest the need for a different management strategy for COVID-19 associated thrombosis. However, clinical trials are necessary to determine the most adequate approach for COVID-19 associated coagulopathy and thrombotic events.

Keywords: COVID-19, blood coagulation disorders, thrombosis.

\section{Introducción}

La infección por el nuevo coronavirus (SARS-CoV-2) ha provocado una de las emergencias sanitarias mundiales más desafiantes de los últimos cien años. Los informes iniciales de la presentación clínica de la enfermedad causada por el SARSCoV-2 (llamada COVID-19) apuntaban hacia una presentación caracterizada predominantemente por enfermedad respiratoria $^{(1)}$. Sin embargo, con el crecimiento exponencial de los casos reportados y las observaciones clínicas realizadas, se reconoció que COVID-19 era una enfermedad compleja con una miríada de manifestaciones clínicas diferentes ${ }^{(2)}$.

Las primeras descripciones del papel del sistema de coagulación en COVID-19 provienen de descripciones de anomalías de laboratorio observadas en estos pacientes, incluyendo elevaciones en los niveles de fibrinógeno y dímero $\mathrm{D}$, y una leve prolongación del tiempo de protrombina (PT) y del tiempo de tromboplastina parcial activada (aPTT), junto con una elevación de los marcadores de inflamación (p. Ej., Proteína C Reactiva) $)^{(3)}$. Posteriormente se reportó un aumento aparente en la frecuencia de eventos trombóticos en pacientes críticamente enfermos con COVID-19 $9^{(4)}$. Estas anomalías parecían semejar a la presentación clínica de la coagulación intravascular diseminada, pero con marcadas diferencias, lo que motivó la descripción de estas anomalías bajo el nombre de coagulopatía asociada a COVID-19. En este artículo procuraremos describir el conocimiento actual de la fisiopatología del rol del sistema de coagulación en COVID19, la presentación clínica de la coagulopatía asociada al COVID-19 y las posibles estrategias para el manejo de esta entidad y sus complicaciones.

\section{Fisiopatología de la coagulopatía asociada a COVID-19}

COVID-19 puede resultar en una enfermedad grave que requiera Cuidados Intensivos (UCI) y ventilación mecánica en el $5-10 \%$ de los pacientes afectados ${ }^{(5)}$. Se ha descrito que estos pacientes críticamente enfermos no solo desarrollan hipoxia, sino que también muestran evidencia de inflamación excesiva y manifestaciones trombóticas que incluyen accidentes cerebrovasculares, embolia pulmonar (EP), trombosis venosa profunda (TVP), trombosis relacionada con catéter y evidencia de trombosis microvascular que afecta los pulmones, riñones, entre otros órganos ${ }^{(4.6-8)}$.

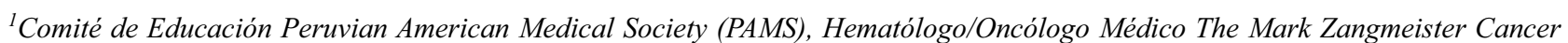
Center, Columbus, Ohio - USA 
Se ha demostrado que el SARS-CoV-2 ingresa a las células mediante la interacción con la enzima convertidora de angiotensina 2 (ECA2) que se expresa en grandes cantidades en el epitelio pulmonar y el endotelio vascular ${ }^{(9)}$. La infección de estas células provoca una alteración de la integridad del epitelio /endotelio con la subsiguiente infiltración de las áreas afectadas por células inflamatorias ${ }^{(10)}$. El infiltrado celular conduce a la producción de altos niveles de citoquinas proinflamatorias como la interleuquina-1 beta (IL-1b), la interleuquina-6 (IL-6) y el factor de necrosis tumoral alfa (TNF-a). Esta respuesta inflamatoria se vuelve excesiva en pacientes críticamente enfermos, lo que conduce a una respuesta inflamatoria sisté$\operatorname{mica}^{(11)}$.

La evidencia actual muestra que el daño endotelial está estrechamente relacionado con el estado de hipercoagulabilidad observado en COVID-19 (Figura 1). La hipoxia que se observa por la afectación pulmonar difusa produce vasoconstricción y reducción del flujo sanguíneo, lo que contribuye aún más a la disfunción endotelial ${ }^{(9,12)}$. También hay un cambio hacia un fenotipo procoagulante del endotelio relacionado con alteraciones de factores de transcripción como el gen 1 de respuesta de grado temprano (Egr-1) y el factor inducible por hipoxia 1 (HIF-1) ${ }^{(6)}$. Este estado proinflamatorio también resulta en una liberación de multímeros del factor von Willebrand ultra grandes (UL-VwF) y sobreexpresión del factor tisular $(\mathrm{TF})^{(13)}$. Las células inflamatorias circulantes se unen al endotelio activado y aumentan la producción de TF y de las trampas extracelulares de neutrófilos (NETs), lo que inicia la coagulación a través de la vía TF/factor VIIa con la consiguiente generación de cantidades excesivas de trombina y el desarrollo de un estado de hipercoagulabilidad ${ }^{(13)}$. Estos fenómenos se ven reforzados por un aumento de los factores procoagulantes como el fibrinógeno y el factor VIII y una posible disminución de los inhibidores de la coagulación como la antitrombina, la proteína $\mathrm{C}$ y la proteína $\mathrm{S}$ como resultado del daño endotelial ${ }^{(13)}$.

La generación excesiva de trombina y el desequilibrio entre los factores pro y anticoagulantes parecen contribuir a la macrotrombosis venosa observada, mientras que la macrotrombosis arterial podría verse afectada por el aumento de los complejos UL-VwF ${ }^{(15)}$. La microtrombosis (la característica distintiva del Síndrome de Distrés Respiratorio Agudo relacionado con COVID-19) parece estar, al menos en parte, relacionada con la alteración de los alvéolos y la microvasculatura pulmonar asociada con complejos ricos en plaquetas y ULVwFs adosados al endotelio dañado junto con el depósito de fibrina intraalveolar formando microtrombos diseminados localizados $^{(16)}$.

\section{Presentación clínica de la Coagulopatía asociada a COVID-19}

Al comienzo de la pandemia, múltiples grupos de investigadores a nivel mundial reportaron diferentes anomalías en las pruebas de laboratorio de pacientes con COVID-19 grave. Estos incluyeron niveles anormalmente altos de

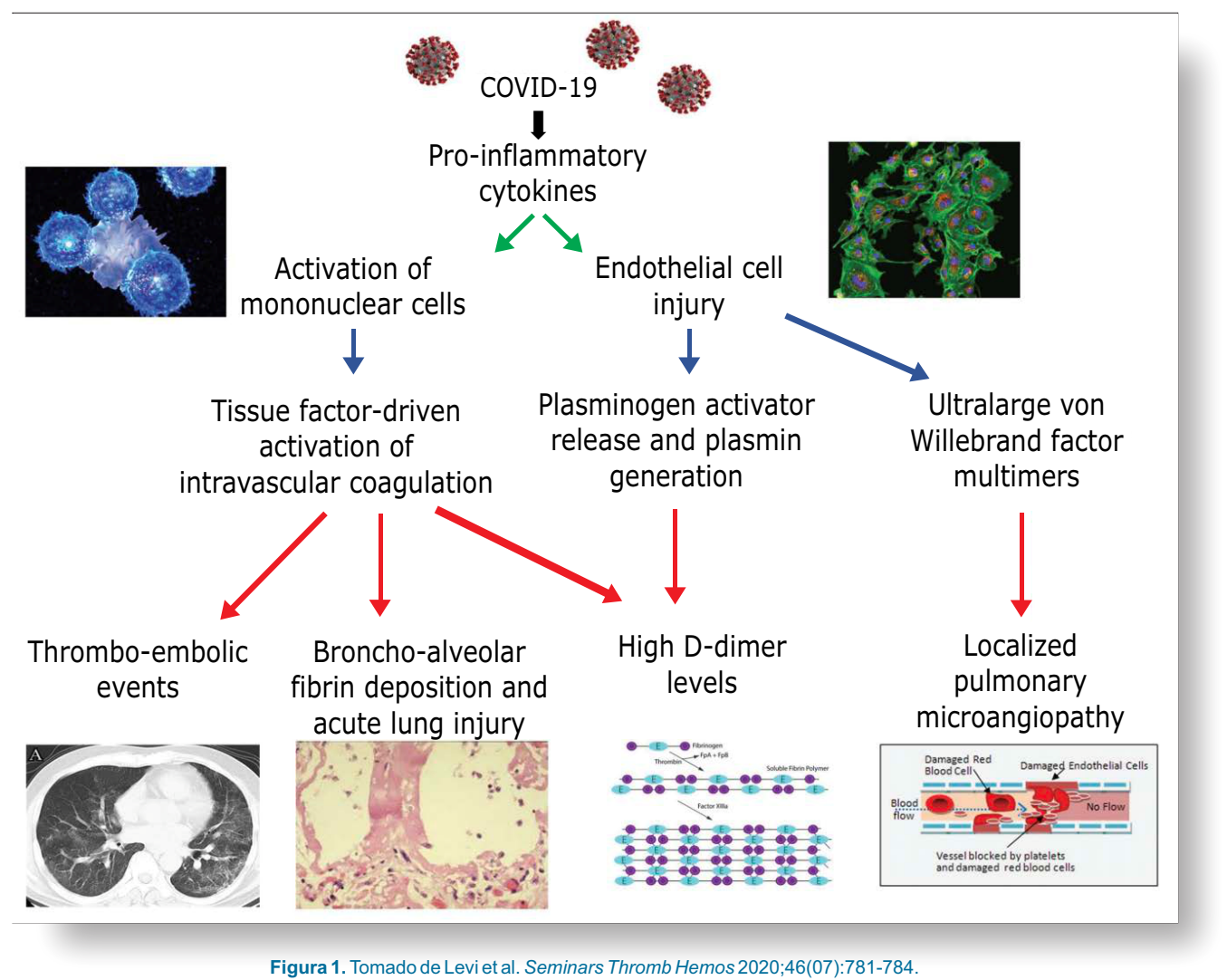


Dímero-D, diversos grados de trombocitopenia, prolongación variable de los tiempos de coagulación y anomalías en los niveles de fibrinógeno, así como disminución de los niveles y actividades de proteína $\mathrm{C}$ y antitrombina ${ }^{(1,2,5,13,17)}$. Estas observaciones condujeron inicialmente a la percepción de que la coagulopatía asociada a COVID-19 podría estar relacionada o parecerse a la coagulación intravascular diseminada (CID). Sin embargo, existen diferencias sutiles pero importantes entre estas entidades (Tabla 1). La mayoría de los pacientes con CID que complica una enfermedad sistémica grave muestran una trombocitopenia profunda ${ }^{(18,19)}$, a diferencia de la trombocitopenia leve que se encuentra en la coagulopatía asociada a COVID19 (en la que los recuentos de plaquetas se observan mayormente entre $100.000 \times 109 / \mathrm{L}$ y $150.000 \times 109 / \mathrm{L}$, con menos del $5 \%$ de los casos presentando recuentos de plaquetas más bajos ${ }^{(1,5)}$. Además, los pacientes con CID tienden a tener niveles mucho más bajos de factores de coagulación y una concentración plasmática de antitrombina y proteína $\mathrm{C}$ muy disminuida, hallazgos que se reflejan en una anomalía más profunda en los tiempos de coagulación, como la prolongación del aPTT o del $\mathrm{PT}^{(20)}$. Además, la coagulopatía asociada a COVID-19 es principalmente protrombótica sin un riesgo significativo de complicaciones hemorrágicas según los datos disponibles $^{(21)}$. Todas estas diferencias se reflejan en el hecho de que la mayoría de los pacientes con coagulopatía asociada a COVID-19 no alcanzarán una puntuación lo suficientemente alta como para ser diagnosticados con CID si se utilizan los criterios de la Sociedad Internacional de Trombosis y Hemostasia (ISTH) para el diagnóstico de CID $^{(22)}$.

Otras entidades protrombóticas, incluyendo microangiopatías trombóticas (MATs) como la púrpura trombocitopénica trombótica o el síndrome urémico hemolítico atípico, comparten algunas características clínicas con la coagulopatía asociada a COVID-19. Sin embargo, no hay pruebas sólidas de trombos microvasculares ricos en plaquetas, que es la característica patológica distintiva de las MATs, en otros órganos además de los pulmones ${ }^{(23)}$. Asimismo, la hemólisis intravascular y la trombocitopenia profunda que se observan típicamente en las MATs, no son características clínicas descritas en los casos graves de COVID-19 $9^{(2)}$.

De todas las anomalías de laboratorio encontradas en pacientes con COVID-19 grave, las elevaciones del Dímero-D fueron una de las primeras descritas y están entre las más intrigantes. Investigadores de China demostraron que las elevaciones del dímero D no solo son frecuentes (ocurren aproximadamente en el $42 \%$ de los pacientes), sino que pueden conllevar información de utilidad pronóstica: en una cohorte retrospectiva de pacientes de Wuhan, China; las elevaciones del Dímero-D se asociaron con un aumento en 18 veces de la mortalidad intrahospitalaria ${ }^{(2)}$. En otra cohorte de pacientes con COVID-19 severo en China, se observaron elevaciones muy altas del Dímero-D (por encima de $500 \mathrm{mg} / \mathrm{L}$ ) en casos fatales. No se encontraron tales elevaciones en los sobrevivientes ${ }^{(3)}$. Estos hallazgos se han replicado en otras partes del mundo ${ }^{(24)}$.

Podría ser que el Dímero-D tenga un papel en el manejo de pacientes con COVID-19 y un impacto potencial en nuestras decisiones de tratamiento: Tang et al. demostraron que la mortalidad por COVID-19 severo aumentó en aquellos pacientes que tuvieron elevaciones del Dímero-D más de 6 veces el límite superior del rango normal. Sin embargo, el riesgo de muerte fue significativamente menor en aquellos pacientes que recibieron dosis profilácticas de heparina en comparación con los que no recibieron este medicamento $(32,8 \% \text { vs } 52,4 \% \text {, OR } 0,44, p=0,017)^{(21)}$.

Otro hallazgo descrito en pacientes con COVID-19 grave fue un aumento en la ocurrencia de eventos tromboembólicos. La primera descripción de estos eventos provino de investigadores en Holanda. Klok et al. reportaron que el 31\% de los pacientes con COVID-19 que requirieron ingreso a la UCI desarrollaron complicaciones trombóticas, incluidos eventos arteriales y venosos ${ }^{(4)}$. Del total de eventos venosos, el embolismo pulmonar fue el más frecuentemente encontrado, abarcando el $81 \%$ de los eventos venosos. Estos hallazgos fueron observados por otros investigadores, quienes describen

\begin{tabular}{|c|c|c|c|}
\hline \multicolumn{4}{|c|}{ Tabla 1} \\
\hline \multicolumn{4}{|c|}{ Coagulopatía por COVID-19 • Coagulación Intravascular Diseminada } \\
\hline & Coagulopatía por COVI-19 & Coagulación Intravascular Disemin. & Microangiopatía Trombótica \\
\hline Plaquetas & $100-150$ & $<100$ & $<100$ \\
\hline $\begin{array}{l}\text { Dimero-D (productos de degradación } \\
\text { de la fibrina) }\end{array}$ & Muy elevado & Elevado & Normal \\
\hline Tiempo de Protrombina (TP) & Normal/Prolongado ( 3s) & Prolongado & Normal \\
\hline Fibrinógeno & Normal/Elevado & Disminuido & Normal \\
\hline Sangrado & Inusual & "Frecuente" & Inusual \\
\hline Hemolisis & Inusual & Frecuente & Frecuente \\
\hline Microangiopatía & Inusual & Frecuente & Frecuente \\
\hline Macrotrombosis & Frecuente (?) & Variable+D15:D18 & Inusual \\
\hline
\end{tabular}

Wang D. JAMA Feb 2020;323(II):1061-9; Zhou. Lancet Mar 2020:395 (I0229): 1054-62 
que los eventos tromboembólicos ocurrieron con mayor frecuencia en el sistema venoso y de aquellos(19), la embolia pulmonar se encontró con mayor frecuencia ${ }^{(7,24,26)}$.

Sin embargo, la frecuencia de estas ocurrencias se ha reportado con una variabilidad significativa en diferentes poblaciones. Por ejemplo, la frecuencia de eventos tromboembólicos venosos en pacientes con COVID-19 que requirieron admisión al hospital pero no atención en UCI oscila entre el 3\% y el $6 \%$ en las diferentes cohortes reportadas. Esto contrasta con los pacientes que requieren tanto ingreso hospitalario como atención en la UCI, en quienes se ha informado la frecuencia de eventos tromboembólicos venosos con un gran grado de variación: entre el $8 \%$ y el $47 \%$ de frecuencia ${ }^{(7,24,27)}$. El riesgo de trombosis y sangrado en pacientes ingresados en el hospital o en la UCI encontrado en algunos de los estudios más grandes disponibles hasta la fecha se resumen en las tablas 2 y 3 . Estos incluyen tanto a pacientes con COVID-19 severo como a pacientes con otros diagnósticos.

Estos reportes han resultado en preguntas importantes que podrían tener un impacto directo en el manejo de pacientes

\begin{tabular}{|c|c|c|c|}
\hline \multicolumn{4}{|c|}{ Tabla 2} \\
\hline \multicolumn{4}{|c|}{ Trombolismo Venoso Sangrado en pacientes hospitalizados (No UCI) } \\
\hline No COVID-19 & Trombo-embolismo Venoso (TEV) & Embolismo pulmonar & Sangrado mayor \\
\hline MEDENOX & $5.50 \%$ & $0 \%$ & $1.70 \%$ \\
\hline PREVENT & $2.80 \%$ & $0.30 \%$ & $0.50 \%$ \\
\hline ARTEMIS & $5.60 \%$ & $0 \%$ & $0.20 \%$ \\
\hline ASH Guidelines & & $0.60 \%$ & $1.90 \%$ \\
\hline COVID-19 & $\begin{array}{l}\text { Eventos thromboticos totales I } \\
\text { Trombo-embolismo Venoso (\%) }\end{array}$ & Embolismo pulmonar (EP) & Sangrado mayor \\
\hline Bilaloglu $(n=2505)$ & $11.5 \% / 4.2 \%$ & $2.20 \%$ & NR \\
\hline Lodigiani $(n=327)$ & $6.4 \% / 3.8 \%$ & $2.50 \%$ & NR \\
\hline Middledorp (n=123) & $3.3 \% / 3.3 \%$ & $1.60 \%$ & NR \\
\hline Al-Samkari $(n=256)$ & $4.7 \% / 3.1 \%$ & $2.70 \%$ & $3.10 \%$ \\
\hline Zhang(Metanalysis) & NR / $6 \%$ & NR & NR \\
\hline
\end{tabular}

NR: No reportado

N Engl J Med 1999; Circulation 2004; BMJ 2006; Blood Adv (2018) 2 (22): 3198-3225; JAMA2020; 325(8):799-801; Thromb Res. 2020;191:9-14; J Thromb Haemos 2020;18:1995-2002; Blood 2020:136(4):489-500; Front. Cardiovasc. Med. 2020;7(151)1:5

\begin{tabular}{|c|c|c|c|}
\hline \multicolumn{4}{|c|}{ Tabla 3} \\
\hline \multicolumn{4}{|c|}{ Tromboembolismo Venoso y Sangrado en pacientes UCI con COVID-19 } \\
\hline No COVID-19 & Trombo-embolismo Venoso (TEV) & Embolismo pulmonar (EP) & Sangrado mayor \\
\hline Kaplan & $37 \%$ & $3.50 \%$ & $1.70 \%$ \\
\hline ASH Guidelines & & $1 \%$ & $4 \%$ \\
\hline COVID-19 & $\begin{array}{l}\text { Eventos thromboticos totales / } \\
\text { Trombo-embolismo Venoso (TEV) }\end{array}$ & Embolismo pulmonar (EP) & Sangrado mayor \\
\hline Bilaloglu $(n=829)$ & $29.4 \% / 15.6 \%$ & $6.20 \%$ & NR \\
\hline Lodigiano $(n=48)$ & $16.7 \% / 8.3 \%$ & $4.20 \%$ & NR \\
\hline Middledorp ( $n=75$ ) & NR / $47 \%$ & $15 \%$ & NR \\
\hline Al-Samkari $(n=144)$ & $18.1 \% / 7.6 \%$ & NR & $5.60 \%$ \\
\hline Zhang(Metanalysis) & NR / 35\% & NR & NR \\
\hline
\end{tabular}

NR: No reportado

Chest 2015 Nov:148(5):1224-1230; Blood Adv (2018) 2 (22): 3198-3225; JAMA 2020; 325(8):799-801; Thromb Res. 2020;191:9-14;

J Thromb Haemos 2020;18:1995-2002; Blood 2020:136(4):489-500; Front. Cardiovasc. Med. 2020;7(151)1:5 
con COVID-19 grave: ¿COVID-19 está intrínsecamente asociado con un mayor riesgo de eventos tromboembólicos conferidos por una biología única de la infección por SARS$\mathrm{CoV}-2$ ? ¿O el aumento percibido en la frecuencia de eventos tromboembólicos es el resultado de la inmovilización, la gravedad de la enfermedad y la necesidad de atención en la UCI en esta población? Investigadores en Francia intentaron responder a estas preguntas. Poissy et al. compararon la frecuencia de EP en pacientes con COVID-19 grave ingresados en la UCI con la frecuencia de EP en un grupo de pacientes ingresados en la misma UCI durante la misma época del año 2019. Encontraron una mayor frecuencia de embolia pulmonar en pacientes con COVID-19 en comparación con los ingresados en la UCI por otras causas $(20,6 \%$ frente a $6,1 \%$; aumento absoluto del riesgo del 14,4\% [IC 95\%, 6,1-22,8]). Estos resultados se mantuvieron incluso después de compararlos solo con pacientes con infecciones distintas al COVID-19 ${ }^{(28)}$. Helms et al. compararon la frecuencia de eventos tromboembólicos en pacientes COVID-19 ingresados en la UCI que desarrollaron Síndrome de Distrés Respiratorio del Adulto (SDRA) con la frecuencia de eventos trombóembólicos en una cohorte histórica de pacientes ingresados en la UCI con SDRA en el mismo centro entre 2014 y 2019. Después de realizar el emparejamiento de puntajes de propensiones, encontraron que los pacientes con COVID-19 tenían una frecuencia significativamente mayor de complicaciones tromboembólicas en total $(11,7$ frente a $2,1 \%, p=0,04)$ asi como de embolias pulmonares $(11,7 \text { frente a } 4,8 \%, p=0,01)^{(6)}$. Los resultados de estos estudios sugieren que podría haber una hipercoagulabilidad única asociada con COVID-19, caracterizada por una fuerte correlación entre la inflamación descontrolada y el desarrollo de eventos tromboembólicos ${ }^{(24)}$.

\section{Manejo de la trombosis asociada a COVID-19}

Si el COVID-19 severo está intrínsecamente asociado con un mayor riesgo de eventos tromboembólicos, se podría plantear la hipótesis de que los pacientes con COVID-19 grave deberían ser manejados con una estrategia de anticoagulación profiláctica de mayor intensidad que el estándar actual, con el fin de reducir el riesgo de estos eventos.

Para que una anticoagulación de mayor intensidad sea beneficiosa, el riesgo de episodios tromboembólicos debería ser considerablemente mayor que el riesgo de hemorragia. $\mathrm{Si}$ bien los datos mencionados anteriormente sugieren que el riesgo de eventos tromboembólicos es mayor en COVID-19, estos datos provienen de estudios observacionales. Existen múltiples fuentes de sesgo inherentes al diseño de los estudios observacionales a tener en cuenta, incluida la confusión por sesgo de indicación, los factores de confusión no medidos (por ejemplo, el riesgo de hemorragia), el efecto de otros tratamientos (Remdesivir, corticosteroides, hidroxicloroquina, azitromicina) en la riesgo de trombosis y sangrado, y el sesgo del tiempo inmortal ${ }^{(29,30)}$. Una mejor estimación del verdadero riesgo de trombosis en pacientes con COVID-19 provendrá de estudios cuyos diseños puedan controlar mejor los sesgos mencionados anteriormente, como los ensayos controlados aleatorios. Muchos de ellos están actualmente en curso y la comunidad científica espera con impaciencia los resultados. Sin embargo, hasta que esos resultados estén disponibles, la comunidad médica debe tomar decisiones a diario en relación al manejo de estos pacientes.

Dada la falta de datos sólidos y resultados de ensayos controlados aleatorios, múltiples instituciones alrededor del mundo han propuesto utilizar una anticoagulación de mayor intensidad para la profilaxis de eventos tromboembólicos en pacientes con COVID-19 (por ejemplo, enoxaparina a $40 \mathrm{mg}$ por vía subcutánea cada 12 horas o heparina no fraccionada a una dosis de 5000 a 7400 unidades internacionales subcutáneas cada 8 horas).

La idoneidad de estas medidas es un tema muy debatido en las comunidades médica y científica en este momento. Las sociedades científicas alrededor del mundo han intentado ofrecer orientación en este tema, dando recomendaciones a veces marcadamente contrastantes (Tabla 4). Sin embargo, parece haber consenso en que, con la información disponible hasta la fecha, la anticoagulación profiláctica a dosis estándar para la prevención de eventos tromboembólicos (en contraposición a la anticoagulación profiláctica de mayor intensidad) parece apropiada para los pacientes ingresados en las salas de hospitalización generales. Sin embargo, dado que la incidencia de eventos tromboembólicos parece ser significativamente mayor en aquellos pacientes con COVID-19 grave que requieren cuidados en UCI, algunas sociedades médicas y científicas han recomendado la anticoagulación de alta intensidad para estos pacientes.

\begin{tabular}{|c|c|c|c|}
\hline \multicolumn{4}{|c|}{ Tabla 4} \\
\hline \multicolumn{4}{|c|}{ Reomendaciones para Anticoagulación Profiláctica en pacientes con COVID-19 } \\
\hline \multirow[t]{2}{*}{ Sociedad Científica } & \multirow{2}{*}{$\begin{array}{l}\text { Pacientes en Hospitalización (No UCI) } \\
\text { Dosis Estándar }\end{array}$} & \multicolumn{2}{|c|}{ Pacientes en $\mathrm{UCl}$} \\
\hline & & Dosis Estándar & Dosis de alta intensidad \\
\hline American Society of Hematology (ASH) & $\checkmark$ & $\checkmark$ & \\
\hline $\begin{array}{l}\text { American College of Chest Physicians } \\
\text { (ACCP) }\end{array}$ & $\checkmark$ & $\checkmark$ & \\
\hline $\begin{array}{l}\text { Int. Society Thrombosis \& Hemostasis } \\
\text { (ISTH) }\end{array}$ & $\checkmark$ & & $\checkmark($ Sugiere) \\
\hline Royal College of Physicians & $\checkmark$ & & $\checkmark($ Considere $)$ \\
\hline
\end{tabular}


Hay muchos informes de efectos persistentes de la infección por COVID-19 después de la infección aguda ${ }^{(31.33)}$. Por lo tanto, se ha planteado la cuestión de si los pacientes con COVID-19 deben recibir anticoagulación profiláctica después de ser dados de alta del hospital. Como ocurre con los pacientes ingresados en el hospital por COVID-19, no hay datos que avalen firmemente ninguna estrategia. Sin embargo, informes recientes parecen sugerir que el riesgo de eventos tromboembólicos y el riesgo de sangrado por anticoagulación después del alta hospitalaria es similar en aquellos con COVID$19 \mathrm{y}$ en aquellos con otras afecciones ${ }^{(34,35)}$.

Si bien la inmensa mayoría de los informes sobre eventos tromboembólicos en COVID-19 provienen de centros hospitalarios con recursos para el diagnóstico y tratamiento de estos eventos; la pandemia también ha afectado a entornos en los que el acceso a pruebas de laboratorio, modalidades de diagnóstico (como el ultrasonido Doppler, la gammagrafía pulmonar de ventilación/perfusión o las angiografías por tomografía computarizada) y las opciones de tratamiento son limitadas. Además, realizar pruebas diagnósticas para eventos tromboembólicos en ocasiones no es factible debido al estado crítico de algunos de estos pacientes (intubación, uso de vasopresores). Por estas razones, algunos líderes de opinión dentro de la comunidad médica han sugerido el uso de anticoagulación "empírica" (administrar dosis completas de anticoagulación sin confirmación objetiva de la trombosis mediante modalidades de imagen) en pacientes con COVID-19 con sospecha de trombosis. Actualmente, sociedades científicas como la Sociedad Americana de Hematología recomiendan considerar la anticoagulación "empírica" como tratamiento en pacientes con COVID-19 grave que tengan sospecha de evento tromboembólico cuando no existan contraindicaciones para la anticoagulación y no exista la posibilidad de realizar estudios de imagen, pero la presentación clínica o los datos de laboratorio (niveles muy altos de dímero D) sugieran convincentemente eventos tromboembólicos y sin otras explicaciones plausibles para los hallazgos clínicos ${ }^{(36)}$.

\section{Conclusiones}

La coagulopatía asociada a COVID-19 parece ser una entidad clínica separada y distinta que resulta del daño endotelial, disfunción y activación del sistema de coagulación impulsado por inflamación incontrolada. Se están realizando múltiples esfuerzos para tratar de aclarar el valor pronóstico y predictivo de los distintos hallazgos clínicos y de laboratorio (elevaciones del dímero D), así como el papel de diferentes estrategias de manejo (anticoagulación profiláctica de mayor intensidad) de pacientes con COVID-19. La comunidad médica espera ansiosamente los resultados de estos esfuerzos, mientras realiza esfuerzos significativos para utilizar los datos disponibles con el fin de servir mejor a nuestros pacientes afectados por esta difícil enfermedad.
Referencias bibliográficas

1. Huang C, Wang Y, Li X, Ren L, Zhao J, Hu Y, et al. Clinical features of patients infected with 2019 novel coronavirus in Wuhan, China. Lancet (London, England). 2020;395(10223): 497-506.

2. Zhou F, Yu T, Du R, Fan G, Liu Y, Liu Z, et al. Clinical course and risk factors for mortality of adult inpatients with COVID-19 in Wuhan, China: a retrospective cohort study. Lancet (London, England). 2020;395(10229):1054-1062.

3. Wang D, Hu B, Hu C, Zhu F, Liu X, Zhang J, et al. Clinical Characteristics of 138 Hospitalized Patients With 2019 Novel Coronavirus-Infected Pneumonia in Wuhan, China. JAMA. 2020;323(11):1061-1069.

4. Klok FA, Kruip M, van der Meer NJM, Arbous MS, Gommers D, Kant KM, et al. Incidence of thrombotic complications in critically ill ICU patients with COVID-19. Thrombosis research. 2020;191:145-147.

5. Guan WJ, Ni ZY, Hu Y, Liang WH, Ou CQ, He JX, et al. Clinical Characteristics of Coronavirus Disease 2019 in China. The New England journal of medicine. 2020;382(18):17081720.

6. Helms J, Tacquard C, Severac F, Leonard-Lorant I, Ohana $\mathbf{M}$, Delabranche $\mathbf{X}$, et al. High risk of thrombosis in patients with severe SARS-CoV-2 infection: a multicenter prospective cohort study. Intensive care medicine. 2020;46(6):1089-1098.

7. Lodigiani C, Iapichino G, Carenzo L, Cecconi M, Ferrazzi P, Sebastian T, et al. Venous and arterial thromboembolic complications in COVID-19 patients admitted to an academic hospital in Milan, Italy. Thrombosis research. 2020;191:9-14.

8. Bikdeli B, Madhavan MV, Jimenez D, Chuich T, Dreyfus I, Driggin E, et al. COVID-19 and Thrombotic or
Thromboembolic Disease: Implications for Prevention, Antithrombotic Therapy, and Follow-Up: JACC State-of-theArt Review. Journal of the American College of Cardiology. 2020;75(23):2950-73.

9. Varga Z, Flammer AJ, Steiger P, Haberecker M, Andermatt R, Zinkernagel AS, et al. Endothelial cell infection and endotheliitis in COVID-19. Lancet (London, England). 2020;395(10234):1417-1418.

10.Zhang W, Zhao Y, Zhang F, Wang Q, Li T, Liu Z, et al. The use of anti-inflammatory drugs in the treatment of people with severe coronavirus disease 2019 (COVID-19): The Perspectives of clinical immunologists from China. Clinical immunology (Orlando, Fla). 2020;214:108393.

11. Tu WJ, Cao J, Yu L, Hu X, Liu Q. Clinicolaboratory study of 25 fatal cases of COVID-19 in Wuhan. Intensive care medicine. 2020;46(6):1117-1120.

12. Liu PP, Blet A, Smyth D, Li H. The Science Underlying COVID-19: Implications for the Cardiovascular System. Circulation. 2020;142(1):68-78.

13. Tang N, Li D, Wang $X$, Sun $Z$. Abnormal coagulation parameters are associated with poor prognosis in patients with novel coronavirus pneumonia. Journal of thrombosis and haemostasis : JTH. 2020;18(4):844-847.

14.Panigada M, Bottino N, Tagliabue $P$, Grasselli G, Novembrino C, Chantarangkul V, et al. Hypercoagulability of COVID-19 patients in intensive care unit: A report of thromboelastography findings and other parameters of hemostasis. Journal of thrombosis and haemostasis : JTH. 2020;18(7):1738-1742.

15. Chang JC. Acute Respiratory Distress Syndrome as an Organ 
Phenotype of Vascular Microthrombotic Disease: Based on Hemostatic Theory and Endothelial Molecular Pathogenesis. Clinical and applied thrombosis/hemostasis : official journal of the International Academy of Clinical and Applied Thrombosis/Hemostasis. 2019;25:1076029619887437.

16. Ciceri F, Beretta L, Scandroglio AM, Colombo S, Landoni G, Ruggeri A, et al. Microvascular COVID-19 lung vessels obstructive thromboinflammatory syndrome (MicroCLOTS): an atypical acute respiratory distress syndrome working hypothesis. Critical care and resuscitation : journal of the Australasian Academy of Critical Care Medicine. 2020;22(2): 95-97.

17.Larsen JB, Pasalic L, Hvas AM. Platelets in Coronavirus Disease 2019. Seminars in thrombosis and hemostasis. 2020;46(7):823-825.

18.George JN, Nester CM. Syndromes of thrombotic microangiopathy. The New England journal of medicine. 2014;371(7):654-666.

19.Akca S, Haji-Michael $P$, de Mendonça A, Suter P, Levi M, Vincent JL. Time course of platelet counts in critically ill patients. Critical care medicine. 2002;30(4):753-756.

20.Gando S, Levi M, Toh CH. Disseminated intravascular coagulation. Nature reviews Disease primers. 2016;2:16037.

21. Tang N, Bai H, Chen X, Gong J, Li D, Sun Z. Anticoagulant treatment is associated with decreased mortality in severe coronavirus disease 2019 patients with coagulopathy. Journal of thrombosis and haemostasis : JTH. 2020;18(5):1094-1099.

22. Taylor FB, Jr., Toh CH, Hoots WK, Wada H, Levi M. Towards definition, clinical and laboratory criteria, and a scoring system for disseminated intravascular coagulation. Thrombosis and haemostasis. 2001;86(5):1327-1330.

23.Fox SE, Akmatbekov A, Harbert JL, Li G, Quincy Brown J, Vander Heide RS. Pulmonary and cardiac pathology in African American patients with COVID-19: an autopsy series from New Orleans. The Lancet Respiratory medicine.2020; 8(7):681-686.

24.Al-Samkari H, Karp Leaf RS, Dzik WH, Carlson JCT, Fogerty AE, Waheed A, et al. COVID-19 and coagulation: bleeding and thrombotic manifestations of SARS-CoV-2 infection. Blood. 2020;136(4):489-500.

25.Bilaloglu S, Aphinyanaphongs Y, Jones S, Iturrate E, Hochman J, Berger JS. Thrombosis in Hospitalized Patients With COVID-19 in a New York City Health System. Jama. 2020;324(8):799-801.
26. Middeldorp S, Coppens M, van Haaps TF, Foppen M, Vlaar AP, Müller MCA, et al. Incidence of venous thromboembolism in hospitalized patients with COVID-19. Journal of thrombosis and haemostasis: JTH. 2020;18(8): 1995-2002.

27.Zhang C, Shen L, Le KJ, Pan MM, Kong LC, Gu ZC, et al. Incidence of Venous Thromboembolism in Hospitalized Coronavirus Disease 2019 Patients: A Systematic Review and Meta-Analysis. Frontiers in cardiovascular medicine. 2020;7:151.

28. Poissy J, Goutay J, Caplan M, Parmentier E, Duburcq T, Lassalle $\mathbf{F}$, et al. Pulmonary Embolism in Patients With COVID-19: Awareness of an Increased Prevalence. Circulation. 2020;142(2):184-186.

29. Kyriacou DN, Lewis RJ. Confounding by Indication in Clinical Research. Jama. 2016;316(17):1818-1819.

30. Maley JH, Petri CR, Brenner LN, Chivukula RR, Calhoun TF, Vinarsky V, et al. Anticoagulation, immortality, and observations of COVID-19. Research and practice in thrombosis and haemostasis. 2020;4(5):674-676.

31. Carfí A, Bernabei R, Landi F. Persistent Symptoms in Patients After Acute COVID-19. Jama. 2020;324(6):603-605.

32. Carvalho-Schneider C, Laurent E, Lemaignen A, Beaufils E, Bourbao-Tournois C, Laribi S, et al. Follow-up of adults with noncritical COVID-19 two months after symptom onset. Clinical microbiology and infection : the official publication of the European Society of Clinical Microbiology and Infectious Diseases. 2020.

33. Garrigues E, Janvier P, Kherabi Y, Le Bot A, Hamon A, Gouze H, et al. Post-discharge persistent symptoms and healthrelated quality of life after hospitalization for COVID-19. The Journal of infection. 2020.

34. Chiasakul T, Evans CR, Spyropoulos AC, Raskob G, Crowther M, Cuker A. Extended vs. standard-duration thromboprophylaxis in acutely ill medical patients: A systematic review and meta-analysis. Thrombosis research. 2019;184:58-61.

35.Patell R, Bogue T, Koshy A, Bindal P, Merrill M, Aird WC, et al. Postdischarge thrombosis and hemorrhage in patients with COVID-19. Blood. 2020;136(11):1342-1346.

36. Hematology ASo. COVID-19 and VTE/Anticoagulation: Frequently Asked Questions 2020 [updated September 18, 2020. Available from: https://www.hematology.org/covid19/covid-19-and-vte-anticoagulation.

Contribución de autoría: Jorge A. Ríos Pérez ha sido autor del artículo, contribuyendo con su concepción, organización de las presentaciones científicas, búsqueda electrónica, revisión inicial, redacción y revisión final. Conflicto de interés: El autor no tiene conflictos de interés con la publicación de este trabajo. Financiamiento: Autofinanciado.

Citar como: El sistema de coagulación y COVID-19. Diagnóstico(Lima). 2020;59(4):187-193.

DOI: 10.33734 /diagnostico.v59i4.254

Correspondencia: Jorge A. Ríos Pérez. Correo electrónico: riosperezjorge@yahoo.com 